\title{
Carbohydrat-fat metabolism disorder in cows and calves
}

\author{
Aleksey Trebukhov ${ }^{*}$, Nadezhda Momot $^{2}$, Yuliya Kolina ${ }^{2}$, Andrey Trebukhov ${ }^{3}$, and Igor \\ Kamliya $^{2}$ \\ ${ }^{1}$ Altai State Agricultural University, 656049 Barnaul, Russian Federation \\ ${ }^{2}$ Primorye State Agricultural Academy, 692510 Ussuriysk, Russian Federation \\ ${ }^{3}$ Altai State University, 656049 Barnaul, Russian Federation
}

\begin{abstract}
Inconsistencies in feeding level of dairy cattle to their feed needs leads to various metabolic diseases, including ketosis. The purpose of our work is to study changes in the metabolism of both sexually mature representatives of dairy cattle and young animals obtained from them. The article states the following causal factors of ketosis occurrence in cows: excessive amount of fat substances, imbalance of various chemical elements in feeding diet. The pathology of carbohydrate-fat metabolism during ketosis in cows was characterized by a significant change in blood biochemical parameters, namely, an increase in triglycerides (up to $0.7 \pm$ $0.07 \mathrm{mmol} / \mathrm{l}$ ), cholesterol (up to $2.7 \pm 0.15 \mathrm{mmol} / \mathrm{l}$ ), non-esterified fatty acids (up to $1.93 \pm 0.19 \mathrm{mmol} / \mathrm{l}$ ), KET (up to $2.4 \pm 0.18 \mathrm{mmol} / \mathrm{l}$ ), low glucose concentration (up to $1.07 \pm 0.12 \mathrm{mmol} / \mathrm{l}$ ) and alkaline reserve (up to $17.8 \pm 1.5 \mathrm{mmol} / \mathrm{l})$. Low level of triglycerides, cholesterol, and alkaline reserve, as well as high values of non-esterified fatty acids, KET, and glucose characterize the exchange in calves from cows with ketosis relative to calves born by cows without metabolic pathology.
\end{abstract}

\section{Introduction}

The development of animal husbandry directly correlates with a healthy animal population, which is supported by a complex of therapeutic and preventive measures $[1,2]$. In modern conditions of market economy, dairy cattle breeding requires high profitability of production. The increase in this indicator is often accompanied by a systematic production intensification, aimed primarily at a regular increase in profits. At the same time, such intensification often outpaces real possibilities of farm intensification in a particular period of time (mismatch of feed base, production culture, zoohygienic parameters, etc.). In such conditions, metabolic pathologies may develop in highly productive animals. [3]. These diseases occur within the framework of the launch of unified adaptation processes, developing simultaneously with the characteristic syndromes of the disease and general, non-specific symptoms noted in various diseases. Pathological change in metabolic processes in the body, prolonged course, absence of clear disease symptoms

\footnotetext{
*Corresponding author: aleks_tav@mail.ru
} 
and often development of latent (subclinical) form of the disease is typical for the development of metabolic diseases, which contributes to these pathologies for a long time to remain in the shadow of the field of vision of farm veterinary specialists [4,5].

The development severity of these pathologies symptoms in high-yielding cattle directly depends on the degree and duration of exposure to etiological factors (diet imbalance in terms of trace elements, lack of particular nutrient, inconsistency of diet nutritional content with level of dairy productivity of animals, insufficient exercise, etc.) [6,7].

Ketosis, osteodystrophy, and other diseases are the most common among these metabolism pathologies in high-producing cows $[8,9]$. These diseases are not only accompanied by a significant decrease in the productivity of livestock, even after recovery, but also significantly reduce the production longevity of animals [10].

Ketone bodies, having no threshold concentration, pass through the blood-placental barrier by diffusion, enter the fetus, trigger intoxication, disorders of normal fetal development, due to changes in metabolism. The result of such an impact is manifested in weak, low-viable young animals [11-13]. The purpose of our work is to study changes in the metabolism of both sexually mature representatives of dairy cattle and young animals obtained from them.

\section{Materials and methods of research}

This study was carried out on the basis of the clinical laboratory of the Faculty of Veterinary Medicine of the Altai State Agrarian University, in the laboratory of the biochemical department of the KSBI "Altai Regional Veterinary Center for the Prevention and Diagnosis of Animal Diseases", and JSC "Uchkhoz "Prigorodnoye" of the city of Barnaul.

The cows of the black-and-white breed, as well as the young animals born from them, were chosen as the object of the study. During the study, two groups of animals were formed - experimental and control. The division into groups was based on the results of the dispensary examination of animals, as well as on the results of urine screening for ketone bodies (KET) in the winter-spring stall-feeding period.

The experimental group consisted of animals with clinical signs of ketosis, while the control group consisted of clinically healthy animals. Similarly, the study groups of young animals from these cows were formed - the experimental group consisted of calves from cows with ketosis, and the control group - calves from cows without signs of ketosis.

In the course of the study, a biochemical blood test was performed in all animals with the determination, according to generally accepted methods [14], of triglycerides (TG), cholesterol (CHL), non-esterified fatty acids (NEF A), glucose (GL) and alkaline reserve(AR) in the blood. The KET content in blood serum and urine was carried out according to the Lestrade method, in a sample with sodium nitroprusside. All biochemical studies were carried out three times. The blood test in cows was carried out according to the scheme - 2 months before calving, 1 month before calving, 10 days after calving, while in calves - it was carried out on the 3rd, 10th, 14th day after birth.

All the data obtained in the work were subjected to statistical processing according to generally accepted formulas, using the StatSoft Statistic software.

\section{Results and discussions}

We carried out a medical examination to determine the clinical status of the herd at the farm, during which we conducted a clinical and laboratory study of 417 heads of dairy herd. Analysis of medical examination results showed that the prevalence of internal diseases in 
the Uchkhoz was $47 \%$ of all diseases diagnosed in the examined cows, and metabolic diseases in the studied cows accounted for $72 \%$ of all established cases of internal noninfectious diseases of animals. In the structure of metabolic pathologies, ketosis (34\%) and osteodystrophy (30\%) in clinical and subclinical forms, diseases of the pancreas $(19 \%)$, liver damage (hepatosis) $(9 \%)$, etc. were most often noted.

Studies of the feeding diet showed its compliance with the standards of the cow needs for basic nutrients. The sugar-protein ratio was - 0.82. At the same time, the analyzed diet showed an excess of fat by $82.6 \pm 9.7 \%$, feed units - by $12.6 \pm 0.8 \%$, metabolic energy by $18.2 \pm 5.5 \%$, and some minerals (iron - by $82.4 \pm 16.8 \%$, calcium - by $9.3 \pm 7.4 \%$, magnesium - by $7.3 \pm 2.6 \%$ ). In the diet of cows, a deficiency of manganese was found - by $26.3 \pm 9.5 \%$. copper by $24.3 \pm 7.8 \%$, cobalt by $22.8 \pm 7.3 \%$, zinc by $6.5 \pm 3.4 \%$, and iodine by $18.6 \pm 7.5 \%$. The calcium-phosphorus ratio was -1.8 .

Biochemical analysis of cow blood that reacted positively to acetone in the urine revealed low sugar content $(2.05 \pm 0.13 \mathrm{mmol} / \mathrm{l})$, alkaline reserve $(16.3 \pm 0.6 \mathrm{mmol} / \mathrm{l})$ and high KET level up to $2.41 \pm 0.15 \mathrm{mmol} / \mathrm{l}$, acetone bodies fractions: dimethyl ketone and acetoacetate $(\mathrm{AcAc})-0.91 \pm 0.06 \mathrm{mmol} / \mathrm{l}$, betta-oxybutyrate $(\mathrm{BHB})-1.56 \pm 0.18 \mathrm{mmol} / \mathrm{l}$. The coefficient demonstrating KET fraction ratio (BHB/AcAc) in the blood amounted $1.71 \pm 0.2$.

Established biochemical changes in cow blood indicate the presence of ketosis, the occurrence of which was facilitated by the revealed non-compliance with the norm of the need for mineral elements in the diet, as well as the excess content of fats in it.

To further study the changes in fat and carbohydrate metabolism during ketosis, we took cows that showed an excess of KET level in the body.

A blood test performed at the 7th month of pregnancy (experimental group) in cows with acetonemia showed a significant change in all the analyzed indicators relative to analogues that do not have signs of the disease. In such a way, the concentration of the indicator was 2 times higher for NEF A, KET - 1.7 times, TG - by $46 \%$, CHL - by $6.5 \%$ in the experimental group of cows.

30 days before calving, the experimental cows showed a decrease in blood content, compared with the initial data: AR by $9.7 \%$ and GL - by $5 \%$. With a simultaneous increase in most of the studied parameters of lipid metabolism, such as TG - by 2 times, NEF A - by $29.5 \%$, KET - by $24 \%$.

In the group of healthy cows, similar dynamics were observed, however, the changes were less pronounced. The GL concentration in blood of animals of this group decreased by $43.4 \%$, NEF A - by $50.5 \%$, AR - by $5 \%$, and TG level, on the contrary, increased by $45.8 \%$, CHL - by $14 \%$, KET - by $16.3 \%$.

The average group values of indicators, 1 month before calving, were higher in the experimental group compared to the control group for NEF A by 3.5 times, TG by 2 times, KET by $21 \%$. While average group values of GL concentration were less in the experimental group for $34 \%$, and AR $-4 \%$.

On the 10th day after calving, the cows of the experimental group showed signs of a deep carbohydrate-fat metabolism disorder. Thus, the GL level in the blood of these animals decreased by $6 \%$ compared to the second study and was also 2 times less than the same control indicator.

The KET and NEF A concentration in the blood of experimental cows significantly increased relative to the second study by $26.4 \%$ and $60 \%$, respectively. In the body of cows without signs of acetonemia, despite the similar dynamics of indicator growth, the increase in NEF A level was only $44 \%$, and KET - by $33 \%$.

The average group values in the control group were still significantly lower in terms of NEF A - 1.7 times, KET - by $13 \%$ relative to the sick animals. 
The CHL concentration in animals with acetonemia decreased by $11 \%$ by the final study, compared to the second study, while TG level significantly decreased by 4 times during this period.

During this period, these indicators in the blood of control cows also decreased, TG content decreased by $20 \%$, and CHL - by $12.4 \%$. In the group of cows with metabolic pathology (experimental), the value of the average group CHL concentration is 1.5 times for TG.

A significant decrease in TG concentration against the background of an increase in NEF A, KET, and a low GL level, in our opinion, indicates a violation of the synthetic liver function in patients with cow ketosis.

The AR concentration in both groups of cows increased slightly by the 3rd study. However, there were no significant differences between the second and third studies in both the experimental and control groups, as well as differences between the values of these average group indicators in the third study.

Table 1. Concentration level of carbohydrate and fat metabolism indicators in the blood serum of cows (mmol/1, M $\pm m, n=34)$

\begin{tabular}{|c|c|c|c|}
\hline \multirow{2}{*}{ Indicator } & $\mathbf{3}$ Concentration \\
\cline { 2 - 4 } & $\mathbf{4}$ & $\mathbf{2}$ & $\mathbf{3}$ \\
\hline \multirow{4}{*}{ Experimental group } \\
\hline KET & $2.4 \pm 0.18$ & $1.82 \pm 0.2$ & $2.3 \pm 0.2$ \\
\hline Cholesterol & $1.97 \pm 0.2$ & $2.7 \pm 0.15$ & $2.4 \pm 0.18$ \\
\hline NEF A & $0.95 \pm 0.07$ & $1.23 \pm 0.1$ & $1.93 \pm 0.19$ \\
\hline Alkaline reserve & $19.9 \pm 1.7$ & $17.8 \pm 1.5$ & $18.3 \pm 1.4$ \\
\hline Triglycerides & $0.35 \pm 0.03$ & $0.7 \pm 0.06$ & $0.18 \pm 0.02$ \\
\hline Glucose & $2.2 \pm 0.13$ & $1.1 \pm 0.1$ & $1.07 \pm 0.12$ \\
\hline \multicolumn{4}{|c|}{ Control group } \\
\hline KET & $1.3 \pm 0.1$ & $1.5 \pm 0.2$ & $2.0 \pm 0.1$ \\
\hline Cholesterol & $1.84 \pm 0.2$ & $2.1 \pm 0.01$ & $1.89 \pm 0.1$ \\
\hline NEF A & $0.44 \pm 0.03$ & $0.35 \pm 0.02$ & $0.5 \pm 0.09$ \\
\hline Alkaline reserve & $19.41 \pm 1.54$ & $18.42 \pm 1.49$ & $19.74 \pm 1.58$ \\
\hline Triglycerides & $0.25 \pm 0.18$ & $0.35 \pm 0.02$ & $0.28 \pm 0.03$ \\
\hline Glucose & $2.95 \pm 0.23$ & $1.67 \pm 0.17$ & $1.3 \pm 0.11$ \\
\hline
\end{tabular}

In accordance with the purpose of the study, a laboratory study of the blood of young cows of both groups was conducted. The results of biochemical study of the blood of young animals are presented in Table 2.

Table 2. Concentration of biochemical parameters of calves' blood ( $\mathrm{mmol} / \mathrm{l}, \mathrm{M} \pm \mathrm{m}, \mathrm{n}=26)$

\begin{tabular}{|c|c|c|c|}
\hline \multirow{2}{*}{ Indicator } & \multicolumn{3}{|c|}{ Concentration } \\
\cline { 2 - 4 } & $\mathbf{1}$ & $\mathbf{2}$ & $\mathbf{3}$ \\
\hline \multicolumn{5}{|c|}{ Experimental group } \\
\hline KET & $0.9 \pm 0.1$ & $1.01 \pm 0.1$ & $1.15 \pm 0.09$ \\
\hline Cholesterol & $1.11 \pm 0.2$ & $1.54 \pm 0.2$ & $0.49 \pm 0.2$ \\
\hline NEF A & $0.37 \pm 0.02$ & $0.55 \pm 0.06$ & $22.3 \pm 2.39$ \\
\hline Alkaline reserve & $20.98 \pm 1.76$ & $21.3 \pm 2.8$ & $0.45 \pm 0.02$ \\
\hline Triglycerides & $0.26 \pm 0.05$ & $0.24 \pm 0.03$ & $5.54 \pm 0.52$ \\
\hline Glucose & $5.0 \pm 0.47$ & $6.13 \pm 0.09$ & $0.82 \pm 0.17$ \\
\hline \multicolumn{2}{|c|}{ Control group } \\
\hline KET & $0.6 \pm 0.05$ & $0.8 \pm 0.1$ & $2.17 \pm 0.2$ \\
\hline Cholesterol & $1.38 \pm 0.2$ & $1.87 \pm 0.2$ & $0.33 \pm 0.02$ \\
\hline NEF A & $0.28 \pm 0.02$ & $0.39 \pm 0.02$ & $23.2 \pm 1.86$ \\
\hline Alkaline reserve & $23.34 \pm 1.53$ & $23.02 \pm 0.86$ & $0.49 \pm 0.05$ \\
\hline Triglycerides & $0.39 \pm 0.04$ & $0.34 \pm 0.02$ & \\
\hline
\end{tabular}




\begin{tabular}{l|l|l|l|} 
Glucose & $3.19 \pm 0.36$ & $5.04 \pm 0.48$ & $6.33 \pm 0.54$
\end{tabular}

Analyzing the obtained data (Table 2), there is a similar dynamics of the concentration values of biochemical parameters in the experimental and control groups of calves, which is expressed in an increase in the concentration of the studied substances by the third study compared to the initial one. At the same time, GL level in the experimental calves, compared with the controls, in the first study, was higher in the blood by $36 \%$ and $17.8 \%$, respectively, and in the third study, on the contrary, lower - by $14.3 \%$.

Analysis of the dynamics of KET concentration in the blood of calves of the experimental group revealed an increase in KET concentration during the entire study period, which by the final stage of the study reached $20 \%$, in comparison with its initial level.

Quantitative analysis of KET content in the blood of calves of the control group also revealed an increase in its level during the study. However, it was significantly lower than the initial KET level in experimental young animals by $18 \%$ by 3 day of life, by 2 weeks after birth (10 days) - by $24 \%$, and by the final study - by $21.5 \%$.

The AR level in the young animals of the experimental group did not fluctuate within large limits during the experiment, and by the end of the observation, it did not have significant differences with the initial level.

Nevertheless, an inter-group comparative analysis showed that its level was significantly lower than that of calves from cows without signs of acetonemia, during the entire experiment. The study of blood biochemical parameters in the experimental group revealed lower TG and CHL levels, and vice versa, high levels of NEF A in comparison with the control.

So, at the beginning of the experiment, TG level of experimental calves was characterized by lower contents than control peers $33.3 \%$, CHL $-20.3 \%$, at the age of 10 days, TG concentration in the group of calves from cows with ketosis was lower by $29 \%$, CHL $-17.6 \%$, while by the 15 th day TG level was lower by $8 \%$, CHL $-16.6 \%$ compared to the control group calves. At the same time, as noted above, the content of NEF A was higher in the blood of calves born from cows with metabolic pathology, relative to that of young control animals: at the beginning of observations - by $32.1 \%$, in the middle (day 10 ) - by $41 \%$ and at the final study (day 14 ) - by $49 \%$.

\section{Conclusions}

1. The main causes of ketosis in dairy cattle were identified - excessive amount of fatty substances in feeding diet and imbalance of various chemical elements.

2. Fat and carbohydrate metabolism disorder in cows reflected in an increase in the blood values of fat (ketone bodies, triglycerides, NEF A, cholesterol) and a significant decrease in the values of carbohydrate (glucose) metabolism.

3. In calves from cows with ketosis, the pathology of carbohydrate-fat metabolism was quantitatively manifested in blood parameters, namely, in the significant increase in NEF A, ketone bodies, glucose, with a simultaneous decrease in triglycerides, alkaline reserve, cholesterol relative to calves born by cows without metabolic pathology.

\section{References}

1. N.V. Momot, Pig breeding, 7, 58 (2020)

2. N.V. Momot, Bulletin of the Altai State Agrarian University, 11(193), 99 (2020)

3. V.G. Ryadchikov, O.G. Shlyakhova, D.P. Dubinina, Scientific Journal of KubSAU, 79, $116(2012)$ 
4. E.N. Tyurenkova, M.T. Moroz, E.A. Oleksiyevych, Major metabolic disorders of highly productive dairy cows (LLC "RC "PLINOR", 2013)

5. D.Sh. Bayteryakov, Scientific notes of KSAVM n.a. N.E. Bauman, 222(2), 21 (2015)

6. A.V. Trebukhov, Ketosis of cows and calves: textbook, 132 (Lan, 2019)

7. A. Trebukhov, A. Elenshleger, IOP Conference Series: Earth and Environmental Science, 341, 012152, (2019)

8. M.E. Ostyakova, Bulletin of KrasSAU, 12, 195-198 (2015)

9. Stengarde L. Swedish University of Agricultural Sciences, 76 (2010)

10. A.V. Trebukhov, Bulletin of the Altai State Agrarian University, 2(136), 125 (2016)

11. A.A. Nikitina, Actual problems of animal husbandry innovative development, 69 (2020)

12. G.G. Mikhin, Izvestiya of the OSAU, 3(41), 109 (2013)

13. A.Elenshleger,A. Lelak, G.Nozdrin,A.Trebukhov, IOP Conference Series: Earth and Environmental Science, 341, 012150, (2019)

14. I.P. Kondrakhin, A.V. Arkhipov, V.N. Levchenko, Methods of veterinary clinical diagnostics: handbook, 520 (KolosS, 2004) 